\title{
Klebsiella variicola
}

National Cancer Institute

\section{Source}

National Cancer Institute. Klebsiella variicola. NCI Thesaurus. Code C86460.

A species of facultatively anaerobic, Gram negative, rod shaped bacteria assigned to the phylum Proteobacteria. This species is nonmotile, oxidase and indole neg ative, and does not ferment adonitol. K. variicola is most commonly isolated from plants but may be an opportunistic pathogen in humans. 